
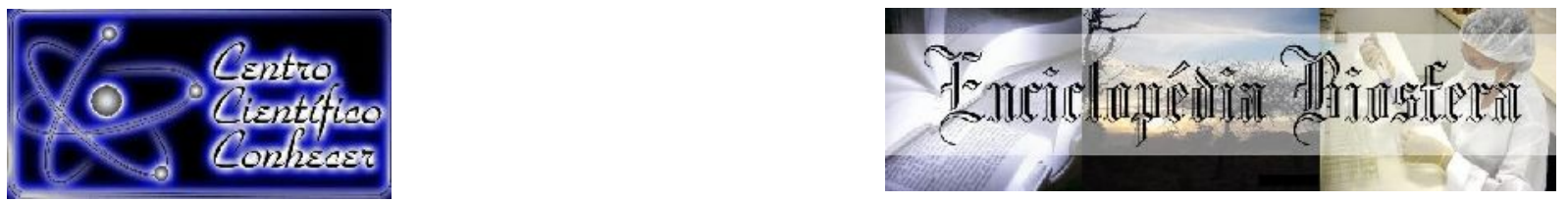

\title{
USO DE INDICES TÉRMICOS PARA ANÁLISE DE REGIÕES PRODUTORAS DE FRANGOS DE CORTE DE MATO GROSSO DO SUL
}

\author{
Natália Coimbra da Silva ${ }^{1}$ Rodrigo Couto Santos ${ }^{2}$ Rafael Zucca ${ }^{3}$ Rafaela Silva Cesca ${ }^{4}$ \\ Luciano Oliveira Geisenhoff ${ }^{5}$
}

1. Tecnóloga em Agroecologia, Doutoranda em Engenharia Agrícola, Faculdade de Engenharia Agrícola, UNICAMP, Campinas-SP, Brasil (n-coimbra@hotmail.com);

2. Engenheiro Agrícola, Professor Doutor da Faculdade de Ciências Agrárias, UFGD;

3. Engenheiro Agrícola, Mestrando em Engenharia Agrícola, Faculdade de Ciências Agrárias, UFGD, Dourados-MS;

4. Engenheira Física, Mestranda em Engenharia Agrícola, Faculdade de Ciências Agrárias, UFGD, Dourados-MS;

5. Engenheiro Agrônomo, Professor Doutor da Faculdade de Ciências Agrárias, UFGD.

Recebido em: 22/09/2018 - Aprovado em: 23/11/2018 - Publicado em: 03/12/2018 DOI: 10.18677/EnciBio_2018B74

\begin{abstract}
RESUMO
O presente estudo teve por objetivo identificar os níveis de estresse em que os frangos de corte possam estar sujeitos durante o período de alojamento utilizando a comparação de dois índices de conforto térmico e analisando condições climáticas históricas de cinco regiões que simulem ambientes distintos no estado de Mato Grosso do Sul (MS). Através de dados climáticos disponibilizados pelo Instituto Nacional de Meteorologia (INMET), referentes às médias diárias de Temperatura (T) e Umidade Relativa do ar (UR) dos meses mais quentes do ano, no período de 20 de dezembro de 2008 a 20 de março de 2017, ano a ano, registrados 24 horas/dia foi verificada a distribuição do Índice de Temperatura e Umidade (ITU) e Entalpia (H) dos municípios de Coxim, Dourados, Paranaíba, Corumbá e Sidrolândia. Com a análise comparativa entre os índices ITU e H, representativos de conforto e analisando condições climáticas históricas, o índice $\mathrm{H}$ demonstrou ser mais rigoroso em identificar os níveis de estresse em que os frangos de corte estão sujeitos durante o período de alojamento.
\end{abstract}

PALAVRAS-CHAVE: Avicultura, Mudanças Climáticas, Variação ambiental.

\section{USE OF THERMAL INDEXES FOR ANALYSIS OF PRODUCTION REGIONS OF BROILER CHICKENS OF MATO GROSSO DO SUL}

\begin{abstract}
The objective of the present study was to identify the stress levels in which broiler chickens may be subjected during their housing period, using a comparison of two thermal comfort indexes and analyzing historical climatic conditions of five regions that simulate different environments in the state of Mato Grosso do Sul (MS). By means of climatic data provided by the National Institute of Meteorology (INMET), referring to the daily averages of Temperature $(T)$ and Relative Humidity $(\mathrm{RH})$ of the hottest months of the year, from December 20 of 2008 to March 20 of 2017, year-on-year, registered 24

ENCICLOPÉDIA BIOSFERA, Centro Científico Conhecer - Goiânia, v.15 n.28; p.907 2018
\end{abstract}


hours a day, the distribution of Temperature and Humidity Index (THI) and Entalpy $(\mathrm{H})$ of the municipalities of Coxim, Dourados, Paranaíba, Corumbá and Sidrolândia were verified. With the comparative analysis between the $\mathrm{THI}$ and $\mathrm{H}$ indices, representing comfort and analyzing historical climatic conditions, the $\mathrm{H}$ index has shown to be more rigorous in identifying the stress levels in which the broilers are subjected during their stay period.

KEYWORDS: Aviculture, Climate changes, Environmental variation.

\section{INTRODUÇÃO}

O Brasil ocupa posições de destaque nos rankings mundiais de produção e exportação de frangos de corte, estando o Mato grosso do Sul entre os sete maiores produtores do país (ABPA, 2018). De acordo com Abreu et al. (2015) o excesso de calor é a principal influência no aumento da taxa de mortalidade, as aves são dependentes de um ambiente adequado para que possam expressar o potencial produtivo, e com isso a manutenção do ambiente térmico, dentro de intervalos de conforto ambiental, torna-se importante para a exploração do potencial da produção.

É indispensável que o animal esteja alojado em um ambiente adequado, para que possa expressar o máximo potencial produtivo, associado ao bem-estar. Assim, o avanço dos sistemas produtivos animais, seja em decorrência do desenvolvimento genético ou da melhoria nas instalações torna o conhecimento de fatores ambientais de extrema importância durante a produção, pois podem refletir na qualidade do produto final (SANTOS et al., 2016).

Segundo De Souza et al. (2015) o bem-estar animal está caracterizado como o estado de harmonia entre este e seu ambiente, por serem sensíveis a elevadas temperaturas e sofrerem inúmeras perdas, não só produtivas, mas também econômicas, principalmente na fase final da produção em consequência do estresse térmico afetando na redução dos índices zootécnicos e no aumento da mortalidade.

Diante disso, torna-se importante conhecer os ambientes de exposição dos animais, como afirmam Jääskeläinen et al. (2014), onde as adequações sofridas por um animal a um ambiente desfavorável também podem alterar a faixa de termoneutralidade, tendo por objetivo sobreviver, se reproduzir e produzir nestas condições extremas.

De acordo com Martins et al. (2016) as variáveis climáticas quando disponibilizadas isoladamente são informações com conteúdo pouco informativo, dada a existência do binômio temperatura ambiente e umidade relativa do ar, influenciadores da variação no desempenho produtivo, pois à medida que a temperatura ambiente aumenta, também compromete as perdas de calor sensível dos animais.

Por envolverem apenas temperatura e umidade relativa do ar os índices Entalpia (H) e o Índice de Temperatura e Umidade (ITU) são bastante utilizados para predizer o desconforto e conforto dos animais, visto que estas variáveis climáticas são disponíveis em quaisquer estações meteorológicas (GUIMARÃES et al., 2014).

Conforme afirmam Machado et al. (2016) o Centro-Oeste brasileiro tem sido um polo promissor na produção de frangos de corte, porém apresenta condições climáticas desfavoráveis, que devem se acentuar com as elevações da temperatura na Terra, ocasionando assim o uso mais intensivo e melhor dimensionado de sistemas de arrefecimento térmico. 
Diante disso, essa pesquisa teve por objetivo utilizar índices de conforto térmico para avaliar diferentes regiões produtoras de aves de corte do estado de Mato Grosso do Sul e verificar se existem respostas diferentes entre a avaliação pelos dois índices diferentes.

\section{MATERIAL E MÉTODOS}

A análise foi realizada no laboratório de Construções Rurais e Ambiência (CRA) da Faculdade de Ciências Agrárias (FCA) da Universidade Federal da Grande Dourados

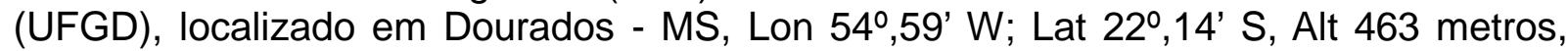
com clima segundo Köppen classificado como Am (clima monçonico, com inverno seco, precipitação média anual de $1.500 \mathrm{~mm}$ e temperatura média de $22^{\circ} \mathrm{C}$ ).

Primeiramente foram buscados dados climáticos coletados por agências oficiais das cidades estudadas, de forma a se ter registrado os diferentes climas do estado de Mato Grosso do Sul em cidades produtoras de aves de corte. Fez-se o uso de dados climáticos disponibilizados pelo Instituto Nacional de Meteorologia (INMET, 2017), referentes às médias diárias de Temperatura (T) e Umidade Relativa do ar (UR) dos meses mais quentes, no período de 20 de dezembro de 2008 a 20 de março de 2017, ano a ano, registrados 24horas/dia, dos municípios de Coxim, Dourados, Paranaíba, Corumbá e Sidrolândia, representando respectivamente o Norte, Sul, Leste, Oeste e Centro do estado.

Os dados foram organizados em planilhas eletrônicas, de modo que cada cidade ficou alocada nas linhas com seus respectivos períodos de observação, e nas colunas foram registradas as variáveis estudadas. A Figura 1 apresenta a área de alcance experimental da pesquisa.

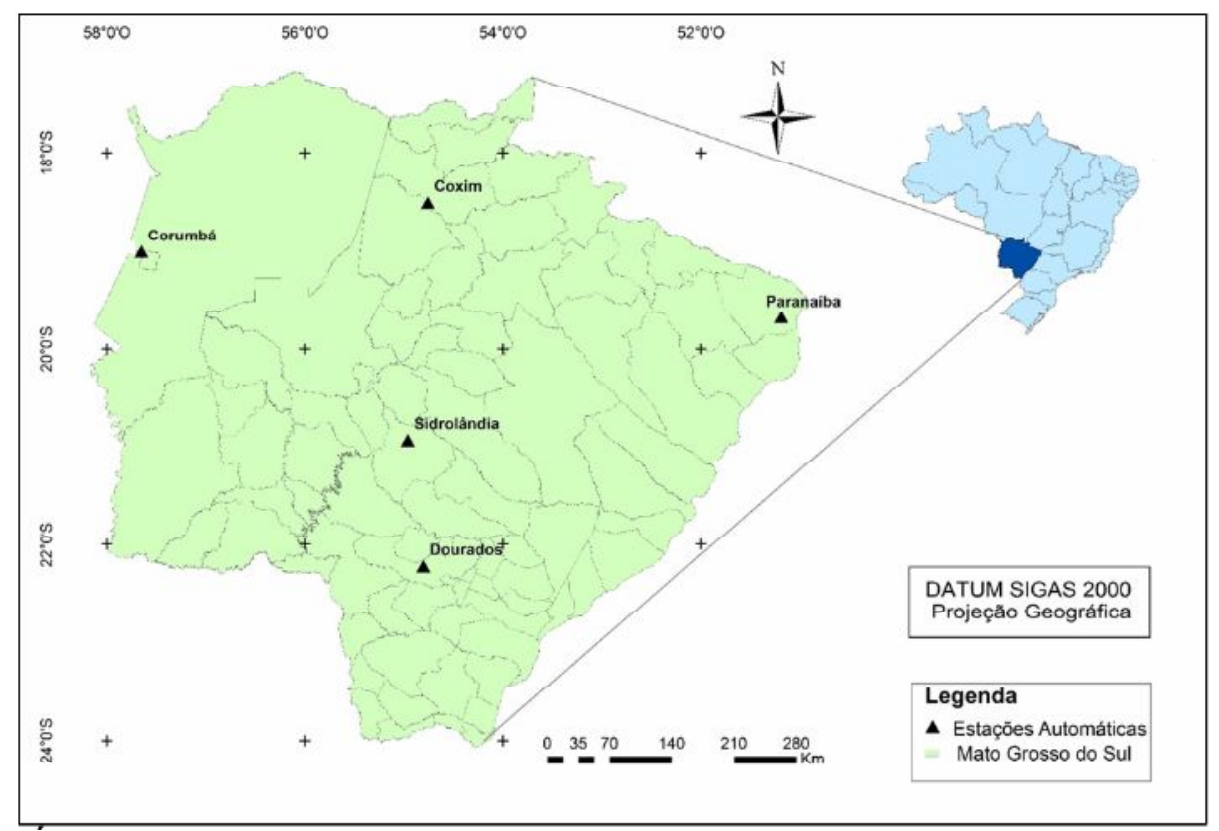

FIGURA 1. Área de alcance experimental dos dados climáticos ressaltados. Fonte: Os autores (2018)

A partir da coleta e organização dos dados, iniciou-se a etapa de tabulação e desenvolvimento da pesquisa utilizando o software MINITAB 17® (MINITAB, 2014). 
Nesta etapa a média das temperaturas máximas e a média das umidades relativas máximas horárias foram correlacionadas para obter-se o valor máximo médio horário dos índices Entalpia $(\mathrm{H})$ e o Índice de Temperatura e Umidade (ITU). Foram calculados os valores de Entalpia $(H)$ para cada período a partir da Equação 1 proposta por Barbosa Filho et al. (2007).

$$
\mathrm{H}=\left(6,7+0,243 * \mathrm{Ta}+\left\{\frac{\mathrm{UR}}{100} * 10 \frac{7,5 * \mathrm{Ta}}{277, \mathrm{Ta}}\right\}\right) * 4,18
$$

\section{Em que:}

$\mathrm{H}$ - Entalpia, $\mathrm{KJ} / \mathrm{Kg}$ ar seco;

Ta - Temperatura do ar, 우

UR - Umidade Relativa do ar, \%.

Os valores indicados de $\mathrm{H}$ na faixa de 54,7 a $62,9 \mathrm{KJ} \mathrm{Kg}^{-1}$ ar seco foram caracterizados como conforto, para valores entre 63,0 a $68,6 \mathrm{KJ} \mathrm{Kg}^{-1}$ de alerta ao estresse térmico, valores entre 68,7 a $75,8 \mathrm{KJ}^{-1} \mathrm{Kg}^{-1}$ como condições de perigo e acima de $75,9 \mathrm{KJ}^{\mathrm{Kg}}{ }^{-1}$ ar seco emergencial, para animais de produção (BARBOSA FILHO et al., 2007). Segundo metodologia de Buffington et al. (1982) utilizando a Equação 2, obteve-se o Índice de Temperatura e Umidade (ITU).

$$
\operatorname{ITU}=0,8 * \mathrm{Ta}+\frac{\mathrm{UR}(\mathrm{Ta}-14,3)}{100}+46,3
$$

\section{Em que:}

ITU - Índice de Temperatura e Umidade, Adm;

Ta - Temperatura do ar, ${ }^{\circ} \mathrm{C}$;

UR - Umidade relativa do ar, \%.

De acordo Gates et al., (1995) para aves de corte, o ambiente é considerado confortável se o ITU for menor ou igual a 74, de alerta se entre 74 e 79 e emergencial à produção se acima de 79 .

\section{RESULTADOS E DISCUSSÃO}

As Figura 2 e 3 mostram as distribuições do Índice de Temperatura e Umidade (ITU) e Entalpia $(H)$, com os índices referentes as cidades de Sidrolândia, Dourados, Coxim, Corumbá e Paranaíba, representando as situações limites de Conforto, Alerta, Perigo e Emergencial para a produção de frangos de corte.

Analisando a Figura 2 observou-se que no período estudado praticamente todas as regiões apresentaram situação de Alerta, com exceção de Dourados que nos verões de 2007, 2008 e 2013, 2014 estavam com médias de ITU confortáveis. Dourados representa o sul do estado, e em uma análise geral teve os menores valores de ITU, 
corroborando com Lima et al. (2014). Já as regiões de Coxim e Corumbá foram as que apresentaram clima mais agressivo se avaliados com base no ITU, sendo Corumbá menos adequado para avicultura, tendo chegado a situação Emergencial no verão 2009, 2010. Pandorfi (2017) afirma que a exposição ao estresse afeta diretamente na redução de produção e reprodução, necessitando melhorar as condições meteorológicas inadequadas das espécies animais, sendo de interesse econômico minimizar a queda na produção.

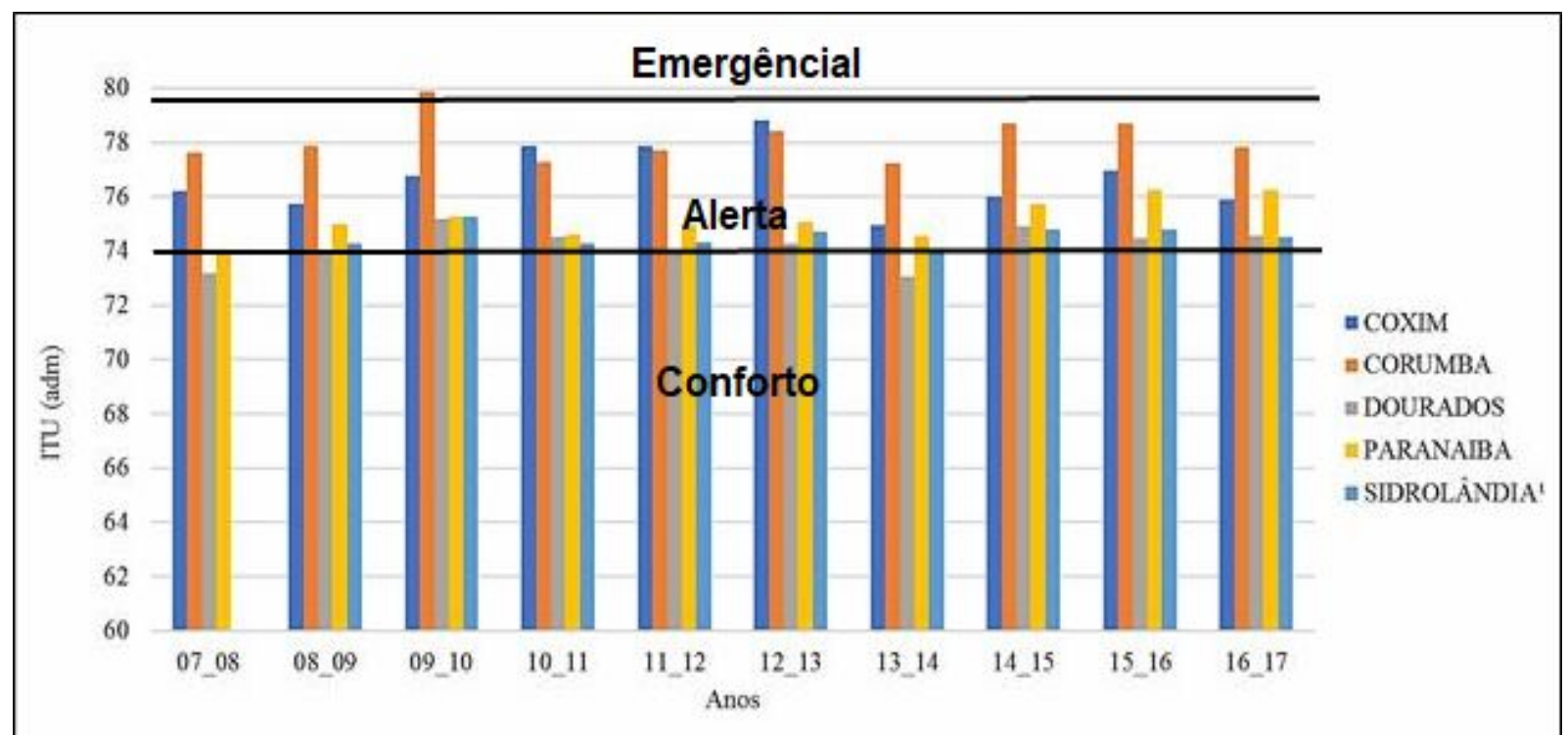

FIGURA 2. ITU médio de verão para o intervalo de 2008 a 2017, ano a ano, para as cidades estudadas.

Fonte: Os autores (2018)

Também é possível observar na Figura 2 que não houve um perfil de aquecimento contínuo com o passar dos anos, resultado este decorrente do curto período de anos usado como amostra. De acordo com Intergovernmental Pannel on Climate Change (IPCC, 2014) por consequência das mudanças climáticas decorrentes do aquecimento global a produção agrícola vem sofrendo com os impactos ambientais e econômicos negativos, sendo necessário estudos mais aprofundados deste fenômeno em regiões produtoras voltadas ao agronegócio.

Ao analisar a Figura 3 é possível observar que para todos os intervalos de verões estudados, a região do município de Corumbá apresentou o maior valor de Entalpia $(H)$, sendo todos no intervalo Emergencial ficando em destaque o verão de 2009,2010 com $79,85 \mathrm{~kJ}_{\mathrm{Kg}}{ }^{-1}$ ar seco, mesmo ano em que o valor de ITU médio também mostrou-se emergencial, atingindo valor de 79,71. Como afirmam Carvalho et al. (2012), a umidade relativa e a temperatura ambiente em conjunto podem ocasionar o aumento da sensação térmica de desconforto atingindo até $50^{\circ} \mathrm{C}$ no interior de uma instalação mal projetada, o que resulta no aumento dos valores de ITU e $\mathrm{H}$, que por sua vez são dependentes de tais variáveis.

Como o índice Entalpia possui uma faixa a mais de conforto é possível observar na Figura 3 que todos os municípios estudados alcançaram a faixa de Perigo à ENCICLOPÉDIA BIOSFERA, Centro Científico Conhecer - Goiânia, v.15 n.28; p.911 2018 
produção e Corumbá também chegou à faixa Emergencial por cinco verões, demonstrando que o índice $\mathrm{H}$ é mais rigoroso na avaliação ambiental, sugerindo que este seja adotado por detalhar mais os níveis de estresse térmico ambiental. Estudos realizados por Pandorfi (2017) demonstram que as alterações climáticas resultam em aumento da temperatura ambiente em algumas regiões brasileiras, devendo ser necessário utilizar métodos para auxiliar na avaliação ambiental e manejo das instalações, de forma a reduzir os impactos do estresse térmico sobre as respostas e o desempenho animal. Segundo DA Guarda et al. (2018) estudar as consequências decorrentes do aquecimento global é um tema que está em evidência em nível mundial, inclusive em se tratando de ambientes construídos.

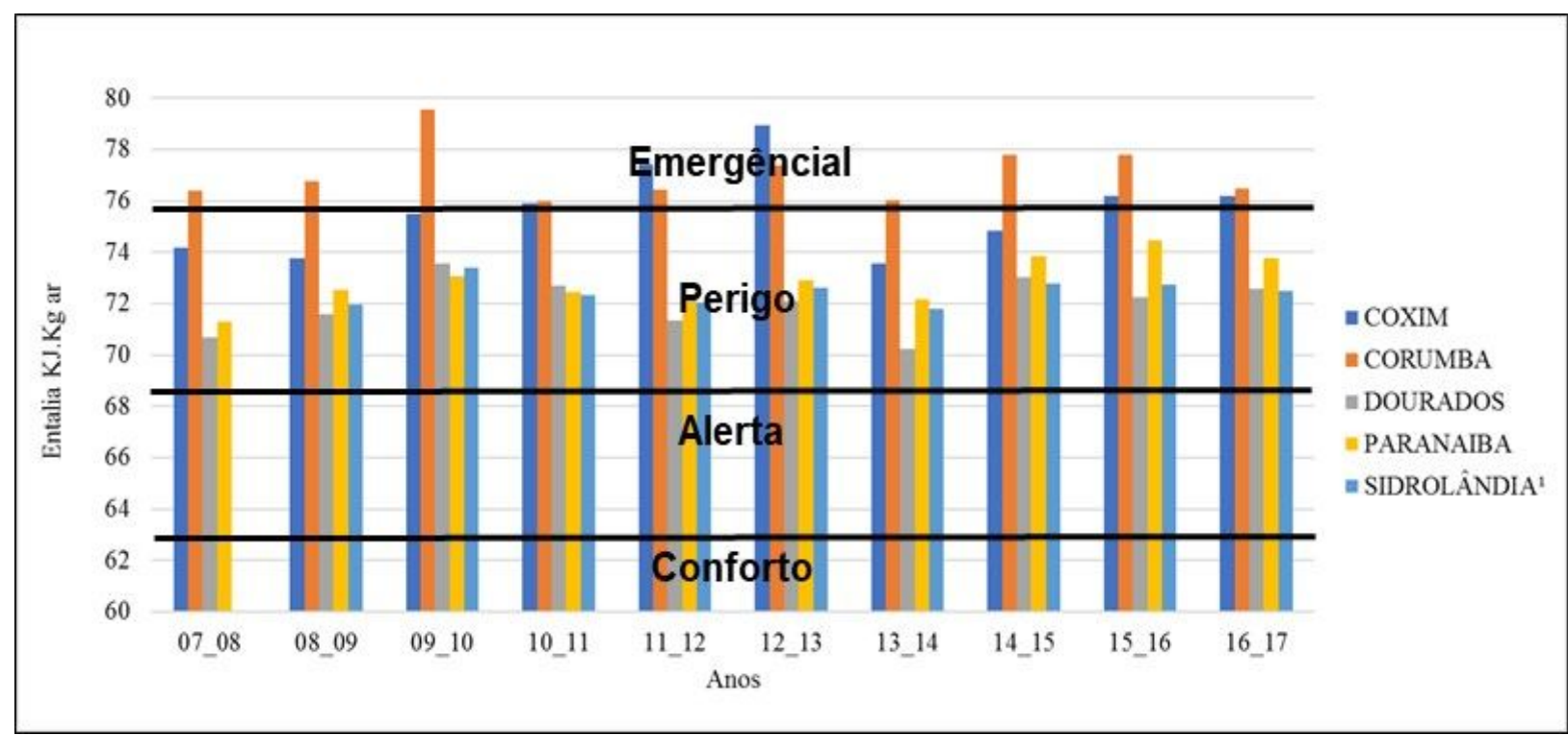

FIGURA 3. Entalpia $(H)$ média de verão para o intervalo de 2008 a 2017, ano a ano, para as cidades estudadas.

Fonte: Os autores (2018)

A principal forma para buscar e executar medidas atenuantes referentes ao desconforto e perda de produção é conhecer o ambiente de exposição, que influencia diretamente no desempenho do animal na forma de estresse térmico (CECCHIN et al., 2016). Assim, verificou-se na Figura 3 que a cidade de Dourados, segundo maior produtor de aves de corte de MS (ABPA, 2018) é a que possuiu melhor clima para a produção de frangos de corte, embora tenha ficado evidente a necessidade do uso de sistemas de arrefecimento térmico, visto que o índice $\mathrm{H}$ esteve sempre acima de 70 $\mathrm{kJ} \mathrm{Kg}^{-1}$ ar seco. O município maior produtor do estado, Sidrolândia, teve sua estação do INMET instalada em 2008,2009 e a partir daí foi possível observar que o ambiente produtivo é intermediário, porém também estressante do ponto de vista dos índices de conforto da Ambiência aqui estudados.

Ao comparar as Figuras 2 e 3 verificou-se que tanto ITU quanto $\mathrm{H}$ demonstraram que de uma maneira geral MS não possui clima confortável para produção para aves de corte em condições de verão. Segundo Queiroz et al. (2017) condições estressantes no interior de um galpão são consequência do ambiente externo e devem ser mitigadas,

ENCICLOPÉDIA BIOSFERA, Centro Científico Conhecer - Goiânia, v.15 n.28; p.912 2018 
sendo necessário um monitoramento eficiente e climatização ambiental adequada em instalações avícolas, para assim obter melhor produtividade final.

\section{CONCLUSÕES}

Com a análise comparativa entre o Índice de Temperatura e Umidade (ITU) e Entalpia $(\mathrm{H})$, representativos de conforto analisando condições climáticas históricas, o índice $\mathrm{H}$ demonstrou ser mais rigoroso em identificar os níveis de estresse em que as aves possam estar sujeitas no período de alojamento.

Observando a análise do ITU e $\mathrm{H}$ médios, utilizando-se as regiões estudadas como referências climáticas no período estudado conclui-se que o sul de Mato Grosso do Sul representado pela cidade de Dourados é a região mais adequada para a atividade avícola por ter um clima mais ameno, sendo que as regiões norte e oeste representadas pelas cidades de Coxim e Corumbá são as de clima mais agressivo, tornando-se necessário maior preocupação com dimensionamento correto do acondicionamento térmico artificial, diretamente relacionado com consumo energético do sistema.

Conclui-se que não há a existência de um padrão nas variações térmicas no período estudado, provavelmente resultado de bolsões de calor e frio, ou curto intervalo de tempo utilizado nesta pesquisa.

\section{AGRADECIMENTOS}

Os autores agradecem à FUNDECT e CNPq pelo apoio financeiro para a concretização desta pesquisa.

\section{REFERÊNCIAS}

ABREU, L. H. P.; YANAGI JUNIOR, T.; FASSANI, E. J.; CAMPOS, A. T.; LOURENÇONI, D. Fuzzy modeling of broiler performance, raised from 1 to 21 days, subject to heat stress. Engenharia Agrícola, Jaboticabal, v. 35 n. 6, p. 967-978, 2015. Disponível em: <http://www.scielo.br/scielo.php?script=sci_arttext\&pid=S0100-69162015000600967>. doi: 10.1590/1809-4430-Eng.Agric.v35n6p967-978/2015.

ABPA - Associação Brasileira de Proteína Animal. A avicultura brasileira. P. 28-65, 2018. Disponível em: <http://abpabr.com.br/storage/files/3678c_final_abpa_relatorio_anual_2017_portugues_web_r eduzido.pdf>. Acesso em: 11 ago. 2018.

BARBOSA FILHO, J. A.; SILVA, I. J.; SILVA, M. A.; \& SILVA, C. J. Avaliação dos comportamentos de aves poedeiras utilizando sequência de imagens. Engenharia Agrícola, v.27, n.1, p.93-99, 2007. Disponível em: <https://doi.org/10.1590/S010069162007000100002>. doi: 10.1590/S0100-69162007000100002.

BUFFINGTON, D. E.; COLLIER, R. J.; CANTON, G. H. Shede managemente systems to reduce heat stress for dairy cows. St. Joseph: American Society of Agricultural engineers, 1982, 16p. (PAPER 82-4061). 
CARVALHO, T.M.R.; MOURA, D.J.; SOUZA, Z.M.; SOURA, G.S.; BUENO, L.G.B.; LIMA, K.A.O. Use of geostatistics on broiler production for evaluation of different minimum ventilation systems during brooding phase. Revista Brasileira Zootecnia, Viçosa, MG, v.41, n.1, p.194-202, 2012. Disponível em: <http://dx.doi.org/10.17224/EnergAgric.2016v31n4p334-341>. doi: 10.17224/EnergAgric.2016v31n4p334-341.

CECCHIN, D.; CAMPOS, A. T.; SCHIASSI, L.; CRUZ, V. M. F. da; SOUSA, F. A. Índice fuzzy para o conforto térmico de suínos na fase de crescimento e terminação com base na temperatura superficial e frequência respiratória. Energia na Agricultura, vol. 31, n.4, p.334-341, 2016. Disponível em: < http://dx.doi.org/10.1590/S151635982012000100028>. doi: 10.1590/S1516-35982012000100028.

DE SOUZA, B. B.; SILVA, R. C.; RODRIGUES, L. R.; RODRIGUES, V. P.; \& DE SOUZA ARRUDA, A. Análises do efeito do estresse térmico sobre produção, fisiologia e dieta de aves. ACSA - Agropecuária Científica no Semiárido, V. 11, n. 2, p. 22-26, 2015. Disponível em: < http://dx.doi.org/10.30969/acsa.v11i2.644>. doi: 10.30969/acsa.v11i2.644.

DA GUARDA, E.L.A.; DURANTE, L.C.; CALLEJAS, I.J.A. Effects of Global Warming on Passive Design Strategies Through Bioclimatic Charts. Engineering and Science, v.7, n.2, p.54-70, 2018. Disponível em: < http://dx.doi.org/10.18607/ES201876827>. doi: $10.18607 / E S 201876827$.

GATES, R. S.; ZHANG, H.; COLLIVER, D. G.; \& OVERHULTS, D. G. Regional variation in temperature index for poultry housing. Transactions of the ASAE, St. Joseph, v.38, n.1, p.197-205, 1995.

GUIMARÃES, M. C. C.; FURTADO, D. A.; NASCIMENTO, J. W. B.; TOTA, L. C. A.; SILVA, C. M.; LOPES, K. B. P. Efeito da estação do ano sobre o desempenho produtivo de codornas no semiárido paraibano. Revista Brasileira de Engenharia Agrícola e Ambiental, v.18, p.231-237, 2014.

INMET. Instituto Nacional de Meteorologia, 2017. Disponível em <http://www.inmet.gov.br/> Acesso em: 20 jun. 2018.

IPCC - Intergovernmental Panel on Climate Change. Climate Change 2014: Impacts, Adaptation, and Vulnerability. Contribution of Working Group II to the Fifth Assessment Report of the Intergovernmental Panel on Climate Change, 2014.

JÄÄSKELÄINEN, T.; KAUPPINEN, T.; VESALA, K. M.; VALROS, A. Relationships between pig welfare, productivity and farmer disposition. Animal Welfare, Washington, v. 23 , p. $435-443$, 2014. Disponível em: <https://doi.org/10.7120/09627286.23.4.435>. doi: 10.7120/09627286.23.4.435.

LIMA, K.A.O.; NAAS, I.A.; GARCIA, R. G.; Impact of Different Light Sources on Broiler ENCICLOPÉDIA BIOSFERA, Centro Científico Conhecer - Goiânia, v. 15 n.28; p.914 2018 
Rearing Environment. Energia na Agricultura, Jaboticabal, v.34, n.3, p.428-434, 2014. Disponível em: <http://dx.doi.org/10.1590/S0100-69162014000300006>. doi: 10.1590/S0100-69162014000300006.

MACHADO, N. S.; TINÔCO, I. F. F.; ZOLNIER, S.; MOGAMI, C. A.; ROCHA, K. S. Aspersão de água de chuva armazenada sobre a cobertura de aviários com telhas de aço na região centro oeste do brasil. Nucleus, v.13, n.1, p. 153-168 abr. 2016. Disponível em: <http://dx.doi.org/10.3738/1982.2278.1556>. doi: 10.3738/1982.2278.1556.

MARTINS, C.L.; OLIVEIRA, D.R.; REIS, E. D.; JUNIOR, R.C.O. Desempenho de frangos de corte submetidos a diferentes sistemas de trocas de ar. Caderno de resumos expandido, p. 152-155, 2016.

MINITAB INC. Minitab® 17. State College, 2014.

PANDORFI, $\mathrm{H}$. Os desafios da ambiência: mudanças climáticas e bem-estar dos animais de produção. In: XLVI Congresso Brasileiro de Engenharia Agrícola (CONBEA). Maceió - AL. 8p, 2017.

QUEIROZ M. L. V.; FILHO, B. J. A.; SALES, F. A. L.; LIMA, L. R. de; DUARTE. L. M. Spatial variability in a broiler shed environment with fogging system. Revista Ciência Agronômica, v.48, n.4, p. 586-595, 2017. Disponível em: <http://dx.doi.org/10.5935/1806-6690.20170068>. doi: 10.5935/1806-6690.20170068.

SANTOS, R. C.; SILVA, S. I.; SILVA, N. C.; BATTILANI, M.; ÁVALO. H.; MARTINS; E. A. S.; DE PAULA, M. O. Análise de risco ao estresse climático na produção de frangos de corte da região do Paraná. In: Simpósio Nacional de Construções Rurais e Ambiência (V SIMCRA/ 2016). Viçosa - MG. p. 16-22, 2016. 